\title{
Article \\ Primaquine Inhibits the Endosomal Trafficking and Nuclear Localization of EGFR and Induces the Apoptosis of Breast Cancer Cells by Nuclear EGFR/Stat3-Mediated c-Myc Downregulation
}

\author{
Ji-Hyang Kim ${ }^{1,2,+}$, Hack-Sun Choi ${ }^{3,4,5,+}$ and Dong-Sun Lee ${ }^{1,2,3,4,5, * \text { (D) }}$ \\ 1 Interdisciplinary Graduate Program in Advanced Convergence Technology \& Science, \\ Jeju National University, Jeju 63243, Korea; seogwi12@jejunu.ac.kr \\ 2 Practical Translational Research Center, Jeju National University, Jeju 63243, Korea \\ 3 Subtropical/Tropical Organism Gene Bank, Jeju National University, Jeju 63243, Korea; choix074@jejunu.ac.kr \\ 4 Faculty of Biotechnology, College of Applied Life Sciences, Jeju National University, SARI, Jeju 63243, Korea \\ 5 Bio-Health Materials Core-Facility Center, Jeju National University, Jeju 63243, Korea \\ * Correspondence: dongsunlee@jejunu.ac.kr \\ + These authors contributed equally.
}

check for

updates

Citation: Kim, J.-H.; Choi, H.-S.; Lee, D.-S. Primaquine Inhibits the Endosomal Trafficking and Nuclear Localization of EGFR and Induces the Apoptosis of Breast Cancer Cells by Nuclear EGFR/Stat3-Mediated c-Myc Downregulation. Int. J. Mol. Sci. 2021, 22, 12961. https://doi.org/10.3390/ ijms222312961

Academic Editor: Anna Kawiak

Received: 8 November 2021

Accepted: 28 November 2021

Published: 30 November 2021

Publisher's Note: MDPI stays neutral with regard to jurisdictional claims in published maps and institutional affiliations.

Copyright: (c) 2021 by the authors. Licensee MDPI, Basel, Switzerland. This article is an open access article distributed under the terms and conditions of the Creative Commons Attribution (CC BY) license (https:// creativecommons.org/licenses/by/ $4.0 /)$.

\begin{abstract}
Triple-negative breast cancer (TNBC) cells overexpress the epidermal growth factor receptor (EGFR). Nuclear EGFR (nEGFR) drives resistance to anti-EGFR therapy and is correlated with poor survival in breast cancer. Inhibition of EGFR nuclear translocation may be a reasonable approach for the treatment of TNBC. The anti-malarial drugs chloroquine and primaquine have been shown to promote an anticancer effect. The aim of the present study was to investigate the effect and mechanism of chloroquine- and primaquine-induced apoptosis of breast cancer cells. We showed that primaquine, a malaria drug, inhibits the growth, migration, and colony formation of breast cancer cells in vitro, and inhibits tumor growth in vivo. Primaquine induces damage to early endosomes and inhibits the nuclear translocation of EGFR. Primaquine inhibits the interaction of Stat3 and nEGFR and reduces the transcript and protein levels of c-Myc. Moreover, primaquine and chloroquine induce the apoptosis of breast cancer cells through c-Myc/Bcl-2 downregulation, induce early endosome damage and reduce nEGFR levels, and induce apoptosis in breast cancer through nEGFR/Stat3dependent c-Myc downregulation. Our study of primaquine and chloroquine provides a rationale for targeting EGFR signaling components in the treatment of breast cancer.
\end{abstract}

Keywords: triple-negative breast cancer; primaquine; nuclear EGFR; Stat3; early endosome; c-Myc

\section{Introduction}

Triple-negative breast cancer (TNBC) lacks estrogen receptor, progesterone receptor, and human epidermal growth factor receptor 2 (HER2) expression and accounts for $15 \%$ to $20 \%$ of breast cancers [1]. TNBC patients have a higher rate of relapse and a worse prognosis than other breast cancer patients [2]. Several research groups have shown that the molecular therapeutic target of TNBC is an epidermal growth factor receptor (EGFR) and that EGFR is overexpressed in TNBC [3,4]. EGFR has two functions: membrane-bound EGFR and nuclear EGFR (nEGFR) [5]. The classic EGFR protein is a cell-membrane-bound receptor tyrosine kinase (RTK) that initiates growth and survival signal pathways [6,7]. Twenty years ago, nEGFR was observed in hepatocytes and was found to be involved in transcriptional regulation, cell proliferation, DNA replication, and chemo- and radioresistance [8,9]. To understand EGFR biology, it is important to study the intracellular trafficking pathway mediating the nuclear translocation of EGFR $[8,10]$. EGF stimulation of plasma-membranelocated EGFR induces the dimerization of EGFR and its internalization into endosomes. Then, EGFR can be translocated into the Golgi/endoplasmic reticulum (ER). EGFR moves 
from the endoplasmic reticulum (ER) into the nucleus [11-14]. nEGFR, as a molecular target in cancer, functions as a cotranscription factor for the expression of several oncogenes: cyclin D1, inducible nitric oxide synthase (iNOS), B-Myb, Aurora kinase A, cyclooxygenase 2 (COX2), c-Myc, breast cancer resistance protein (BCRP), and signal transducer and activator of transcription 1 (Stat1) [15-21]. nEGFR activates DNA-dependent protein kinase (DNA-PK) to enhance DNA repair [22]. These data suggest that tumors are dependent on two distinct compartments of EGFR signaling to give oncogenic properties: membranebound EGFR signaling and nEGFR signaling [5]. Cancer cells that are resistant to cetuximab, a monoclonal antibody that binds to EGFR, express high levels of nEGFR and Src family kinase (SFK) [23,24]. The SFK-dependent phosphorylation of EGFR, at tyrosine 1101, plays an important role in the initiation of EGFR nuclear translocation [25]. EGFR is a therapeutic target in cancer patients. However, previous studies have shown that TNBC is resistant to the EGFR-targeting antibody cetuximab or EGFR inhibitors (tyrosine kinase inhibitors, TKIs). The nuclear translocation of EGFR reduces the inhibitory effects of cetuximab and confers AXL RTK-mediated cetuximab resistance through the activation of downstream signaling $[5,10]$.

The internalization of ligand-induced EGFR is mediated by clathrin- and lipid-raftmediated endocytosis [26]. Inhibition of EGFR endocytosis decreased the proliferation and induced the apoptosis of cancer cells. Rab25 plays a critical role in EGFR endocytosis. EGFR endocytosis is a novel therapeutic target in cancer with wild-type EGFR [27].

In this study, primaquine, a malaria drug, was found to induce early endosome damage and reduce nEGFR; primaquine was also found to induce apoptosis levels in breast cancer through nEGFR/Stat3-dependent c-Myc downregulation. Our data suggest that primaquine can be used in breast cancer treatment as it targets the nuclear translocation of EGFR.

\section{Results}

2.1. The Antimalarial Drug Primaquine Decreases Breast Cancer Cell Viability and Inhibits Tumor Growth in a Mouse Xenograft Model

To examine the effect of primaquine on the viability of TNBC cells, MDA-MB-231 cells and HCC1937 cells were investigated by a 3-(4,5-Dimethylthiazol-2-yl)-5-(3-carboxymethoxyphenyl)-2-(4-sulfophenyl)-2H-tetrazolium (MTS) assay, which detects cell proliferation. Compared to those with dimethyl sulfoxide (DMSO), the live breast cancer cells incubated with primaquine were significantly decreased $\left(\mathrm{IC}_{50}: 81.2 \mu \mathrm{M}\right)$ (Figure $1 \mathrm{~A}, \mathrm{~B}$ and Supplementary Figure S1). However, primaquine showed no inhibitory effect on the proliferation of MCF-7 (ER+) and MDA-MB-453 (HER2+) cells (Figure 1B). Next, we assayed the effects of primaquine on cell migration and colony formation. Primaquine reduced the cell migration and colony formation of breast cancer cells (Figure 1C,D). These results suggest that primaquine directly controls cell growth and biological behaviors in breast cancer. Based on the inhibitory effects of primaquine on cell proliferation, we examined the effect of primaquine on tumor growth in a xenograft model of breast cancer. As shown in Figure $1 \mathrm{E}$, tumor-bearing mice were treated with or without primaquine $(2 \mathrm{mg} / \mathrm{kg})$ once every 10 days for 90 days. Primaquine treatment inhibited tumor growth, as indicated by a decrease in the volume of the observed tumors (Figure 1E). Mice in the primaquine-treated group and control group showed similar body weights (Figure 1E). Our data indicates that primaquine inhibits tumor growth in a xenograft mouse model. 
A

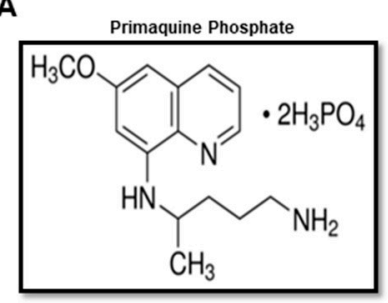

C

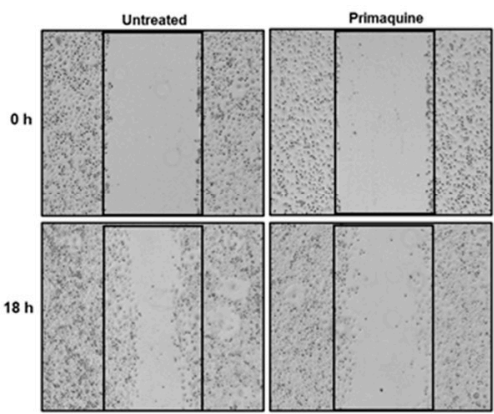

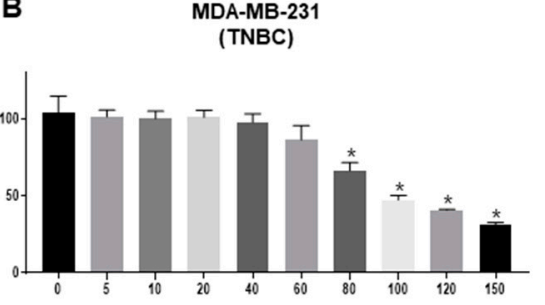

D
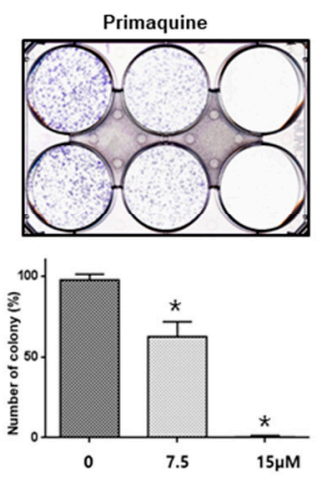

E
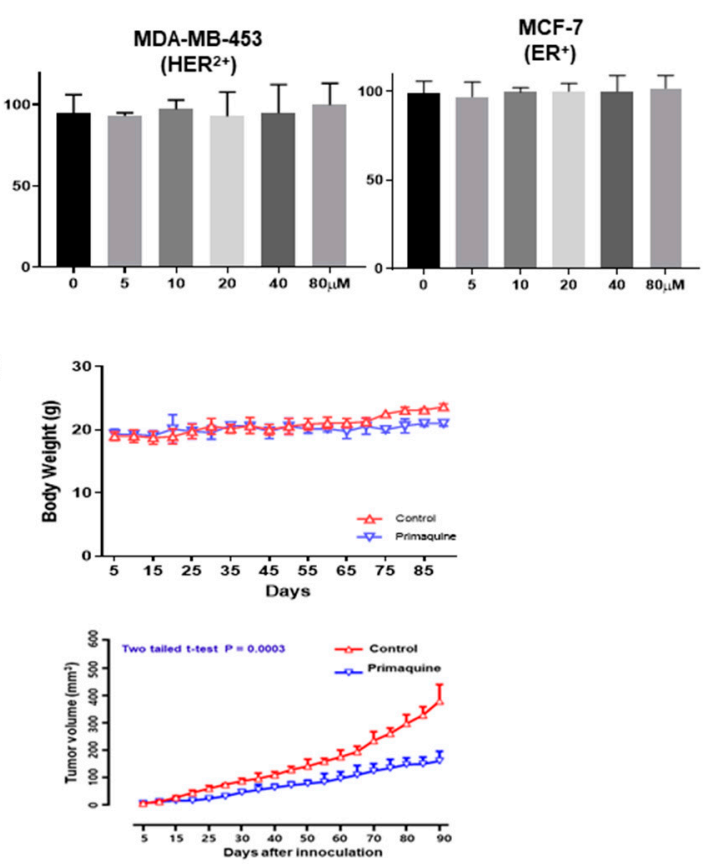

Figure 1. Effect of primaquine on breast cancer hallmarks and on tumor growth in a nude mouse model. (A) Molecular structure of primaquine phosphate. (B) The proliferation of breast cancer cells was measured using an MTS assay kit and the CellTiter 96 Aqueous One Solution kit. Breast cancer cells were incubated in 96-well plates in the presence of primaquine $(5,10,20,40,80,100,120$, and $150 \mu \mathrm{M}$ and DMSO). Values are the mean \pm standard deviation (SD) of 3 independent experiments. ${ }^{*}$ indicates $p<0.05$ vs. control. (C) Effect of primaquine on the migratory ability of breast cancer. The migration of MDA-MB-231 cancer cells with/without primaquine was photographed at 0 and $18 \mathrm{~h}$. (D) Effect of primaquine on the colony formation of breast cancer cells. Two thousand MDA-MB-231 cells were cultured in 6-well plates with/without primaquine at the indicated concentrations for one week. Representative image of colonies. Values are mean \pm SD of 3 independent experiments. * indicates $p<0.05$ vs. control. (E) The effect of primaquine on tumor growth in a xenograft mouse model. A total of $2 \times 10^{6}$ cancer cells were injected into the mammary fat pad of each nonobese diabetic/severe combined immunodeficiency (NOD/SCID) female nude mouse. Effect of tumor growth on primaquine and MDA-MB-231 cell-bearing immunodeficient nude mice. The dose of drug used was $2 \mathrm{mg} / \mathrm{kg}$ once every 10 days. Tumor volume was measured once every 10 days using a caliper and calculated as (width ${ }^{2} \times$ length) $/ 2$. Tumor growth curves were monitored during the experimental period.

\subsection{Primaquine Affects the Endolysosomal System and Impairs the Endocytosis-Mediated Degradation of EGFR}

As a malaria drug, chloroquine (CQ) can affect the function of the endolysosomal system and impair the endocytosis-mediated degradation of EGFR [28]. We assessed the early endosome protein marker EEA1 upon treatment with primaquine by immunofluorescence microscopy. When MDA-MB-231(TNBC) and MDA-MB-453 (HER2+) cells were exposed to primaquine (Figure 2A,B), the distribution of EEA1 on MDA-MB-231 cells was changed, and its signal became faint over time. However, the distribution of EEA1 (early endosome marker) on MDA-MB-453 cells was not changed. Our results showed that primaquine alters the endolysosomal system of TNBC cells. In the control cells, EGFR was rapidly internalized following EGF treatment, and primaquine treatment decreased the EGFR internalization rate after 15 min of EGF treatment (Figure 2B). Primaquine did not changed EGFR internalization of EGF treatment on MDA-MB-453 cells (HER2+), but it changed EGFR internalization of EGF treatment on MDA-MB-231 cells (TNBC). Our immunofluorescence staining of EGFR showed its perinuclear accumulation in Figure 2B. This data showed disrupted endocytic trafficking. We showed that primaquine dysregulated the endolysosomal system and EGFR endosomal trafficking, and induced the endocytosis-mediated degradation of EGFR in TNBC cells. 

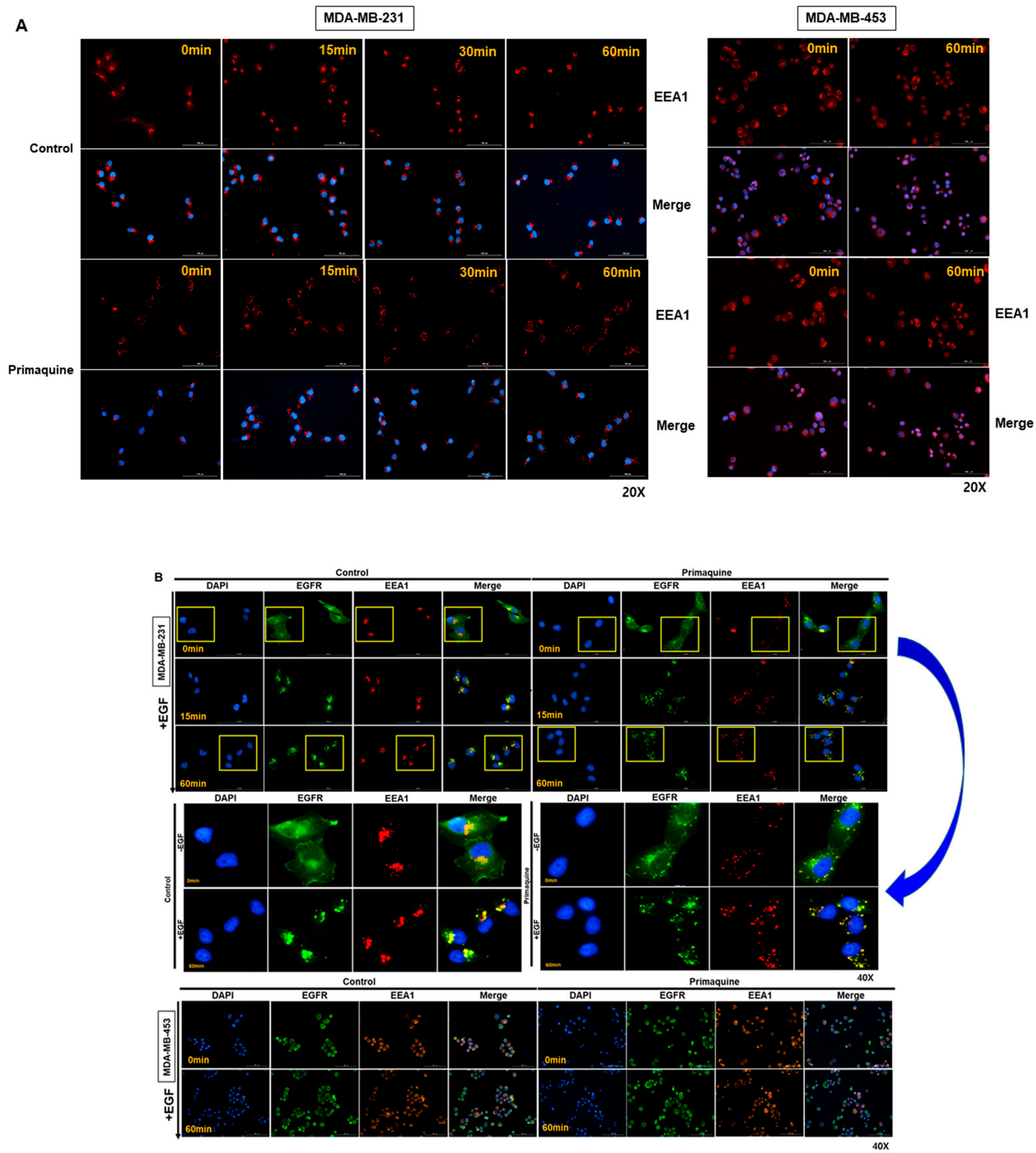

Figure 2. Primaquine has an impact on the endosome system and impairs the endocytosis-mediated degradation of EGFR. (A) MDA-MB-231 and MDA-MB-453 cells were exposed to DMSO or $50 \mu \mathrm{M}$ primaquine for $1 \mathrm{~h}$ before processing for immunofluorescence microscopy and stained with anti-EEA1 and DAPI. (B) MDA-MB-231 and MDA-MB-453 cells were exposed to $50 \mu \mathrm{M}$ primaquine or DMSO for 1 day and then treated with EGF from 0 to 60 min. Before processing for immunofluorescence microscopy, cancer cells were stained with anti-EEA1 and anti-EGFR. Insets represent high magnification images of the region indicated by the yellow rectangle. 


\subsection{Primaquine Reduces the Expression of $n E G F R$ in Breast Cancer}

Breast cancer cells overexpress EGFR, and EGFR has two functions: membrane-bound signaling and nuclear signaling. nEGFR enhances resistance to anti-EGFR therapies and is a functional molecular target in TNBC [5]. As primaquine impairs the endocytosismediated degradation of EGFR, we assessed nEGFR expression in breast cancer. MCF-7 $(\mathrm{ER}+)$ and MDA-MB-453 (HER2+) cells did not show nEGFR expression according to Western blotting (Figure 3B). MDA-MB-231 cells exhibited nEGFR expression according to immunofluorescence microscopy and Western blotting (Figure 3A,B). After treatment with primaquine, we again examined nEGFR expression in breast cancer cells. The levels of nEGFR were decreased in a primaquine-concentration-dependent manner (Figure 3B), and lower levels of nEGFR were also confirmed by immunofluorescence microscopy (Figure 3C). We examined the localization of EGFR after treatment with primaquine on MDA-MB-231 and HCC-1937 cells; primaquine reduced the cytosolic and nuclear fraction of EGFR and pEGFR (Figure 3B and Supplementary Figure S1C).

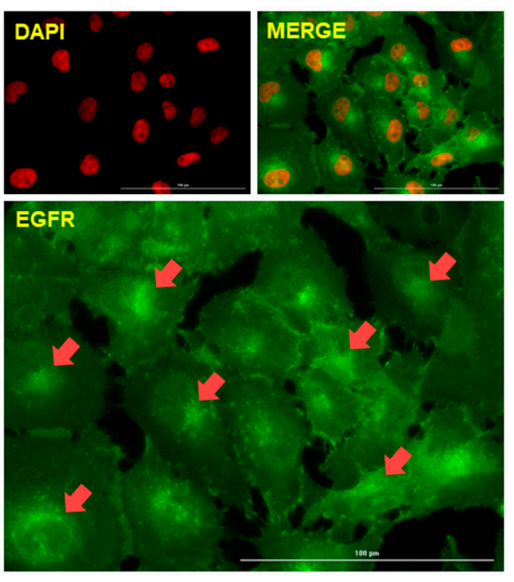

MDA-MB-231

C
B

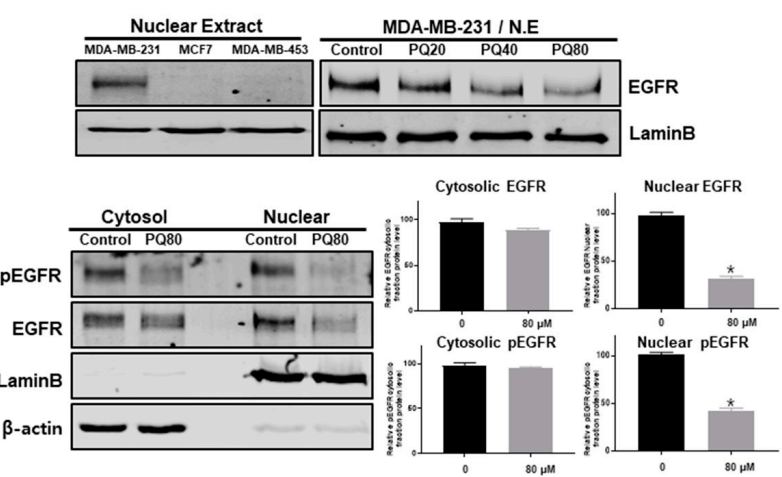

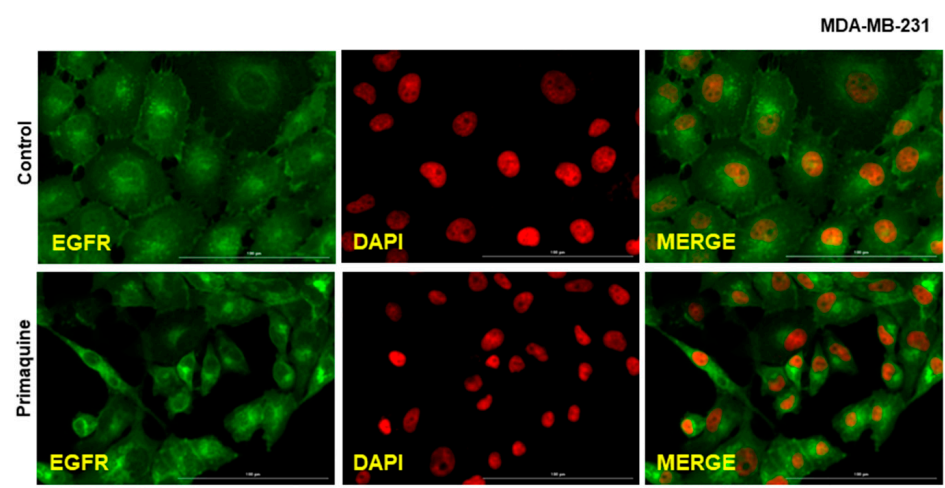

Figure 3. nEGFR expression was reduced in MDA-MB-231 cells upon primaquine treatment. (A) Nuclear localization of EGFR with anti-EGFR (green) and nuclei (red, DAPI) using a fluorescence microscope (Lionheart FX, BioTek, Winooski, VT, USA). nEGFR resulted from merging of EGFR (green) and DAPI (red). (B) MCF-7, MDA-MB-453, and MDA-MB-231 cells were treated with primaquine $(20,40$, and $80 \mu \mathrm{M})$ for $24 \mathrm{~h}$ and subjected to Western blot analysis. Cells were finally lysed, and cytosolic and nuclear proteins were isolated. The cytosolic and nuclear proteins were identified with anti-pEGFR and anti-EGFR antibodies by Western blotting. The expression of lamin B and $\beta$-actin were determined as a loading control for nuclear and cytosolic lysates. Values are the mean \pm SD of 3 independent experiments. * indicates $p<0.05$ vs. control. (C) Cancer cells with/without primaquine were washed, fixed, permeabilized, and blocked with $0.1 \%$ normal goat serum for $60 \mathrm{~min}$. Cells were incubated with primary monoclonal EGFR antibody. Immunostained cells were examined with a fluorescence microscope (Lionheart FX, BioTek, Winooski, VT, USA). Red, nuclei stained by DAPI; green, EGFR. nEGFR was identified by merging EGFR (green) and DAPI (red) images. 


\subsection{Primaquine Regulates EGFR Phosphorylation and the EGFR Downstream Signaling Pathway}

To assess the effect of primaquine on EGFR phosphorylation and the EGFR downstream signaling pathway, we analyzed the activity of EGFR and its downstream signaling proteins, such as ERK and Stat3. Primaquine treatment combined with EGF exhibited a prolonged increase in EGFR phosphorylation compared to EGF treatment alone (Figure 4A). Although the autophosphorylation of EGFR disappeared over time in the control, the turnover of pEGFR was delayed in primaquine-treated cells (Figure 4A). To confirm EGFR downstream signaling pathways, breast cancer cells were treated with primaquine. Primaquine treatment was involved in regulating Stat3 signaling but not ERK signaling (Figure $4 B, C$ ). These data reveal that the combination of EGF and primaquine treatment in breast cancer resulted in a prolonged increase in EGFR phosphorylation. Primaquine might dysregulate EGFR signaling and downregulate the Stat3 signaling pathway.

A
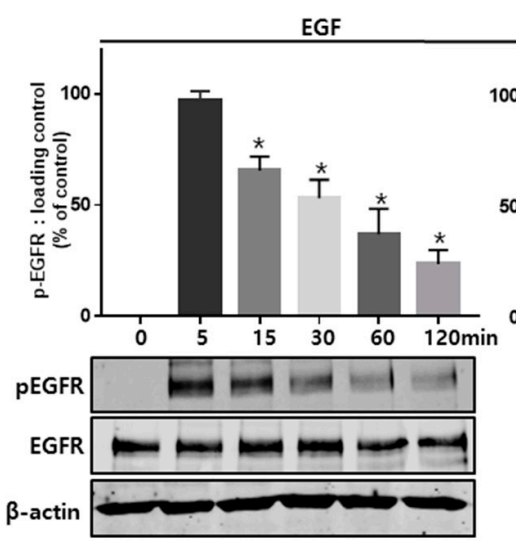

B

$\mathrm{EGF}+\mathrm{PQ} 50 \mathrm{uM}$

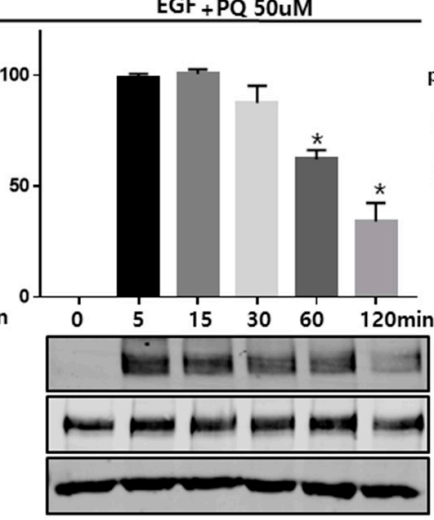

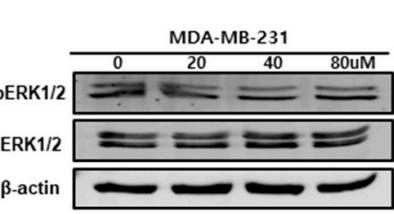

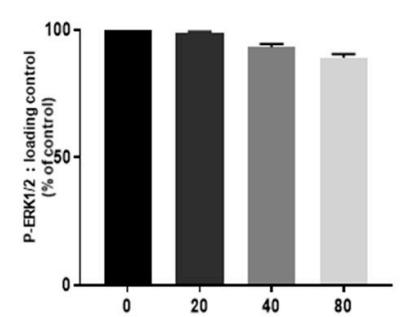

C
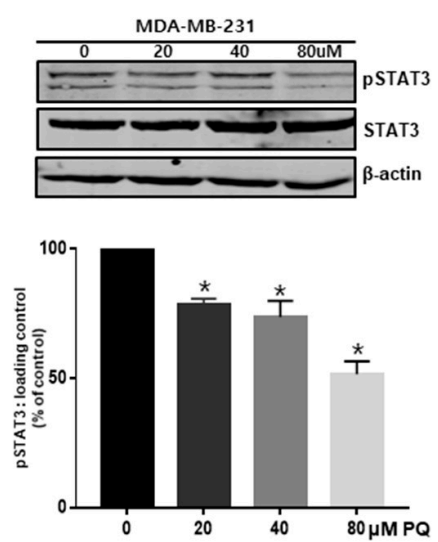

Figure 4. Primaquine treatment delayed EGFR signaling and reduced Stat3 signaling. (A) MDA-MB-231 cells were exposed to DMSO or $50 \mu \mathrm{M}$ primaquine for $24 \mathrm{~h}$ and then treated with EGF from 0 to $120 \mathrm{~min}$. Cells were finally lysed and proteins were identified with anti-pEGFR and anti-EGFR antibodies by Western blotting. (B) MDA-MB-231 cells were exposed to DMSO or 20, 40, and $80 \mu \mathrm{M}$ primaquine for $24 \mathrm{~h}$. Cancer cells were finally lysed and proteins were identified with anti-pERK1/2 and anti-ERK1/2 antibodies by Western blotting. (C) MDA-MB-231 cells were exposed to DMSO or 20, 40, and $80 \mu \mathrm{M}$ primaquine for $24 \mathrm{~h}$. Cancer cells were finally lysed and proteins were identified with anti-pStat3 and anti-Stat3 antibodies by Western blotting. The data are presented as the mean $\pm \mathrm{SD} ; n=3$; $^{*}$ indicates $p<0.05$ vs. control.

\subsection{The nEGFR Protein Physically Interacts with Stat3 and this Interaction Is Inhibited by Primaquine Treatment}

As EGFR signaling regulates Stat3 phosphorylation, we assessed the interaction of nEGFR and nuclear Stat3. Immunoprecipitation using anti-EGFR and anti-Stat3 antibodies showed that nEGFR interacts with nuclear Stat3 (Figure 5A). Primaquine inhibited the interaction of Stat3 and EGFR. The nEGFR/Stat3 complex is known to regulate the transcription of the Stat1, Aurora A, COX2, and c-Myc genes (Figure 5B,E) [29]. We assessed the mRNA levels of these four genes under primaquine treatment using RT-qPCR, and only the transcript levels of c-Myc were decreased (Figure 5C). We confirmed that the total and nuclear c-Myc protein levels were decreased by primaquine treatment through Western blot analysis using an anti-c-Myc antibody (Figure 5C). To confirm that nuclear Stat3 regulates c-Myc, we downregulated the Stat3 gene using si-Stat3. Stat3 downregulation induced a decrease in the c-Myc gene (Figure 5D). Our data showed that primaquine decreases c-Myc gene expression by inhibiting the formation of the Stat3/nEGFR complex (Figure 5E). 
A

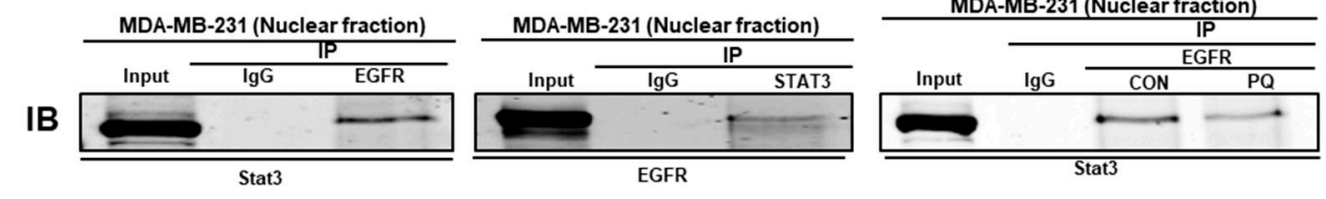

B

Target genes of Stat3/nEGFR: Stat1, Aurora-A, COX-2 and c-Myc

C
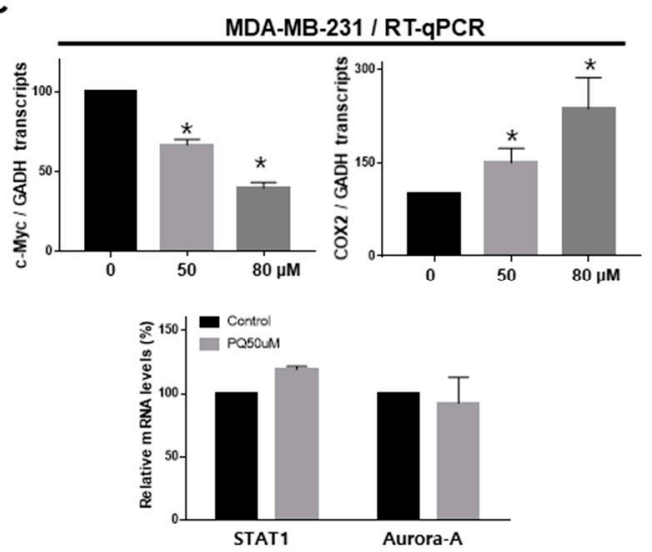

MDA-MB-231 / Western blotting
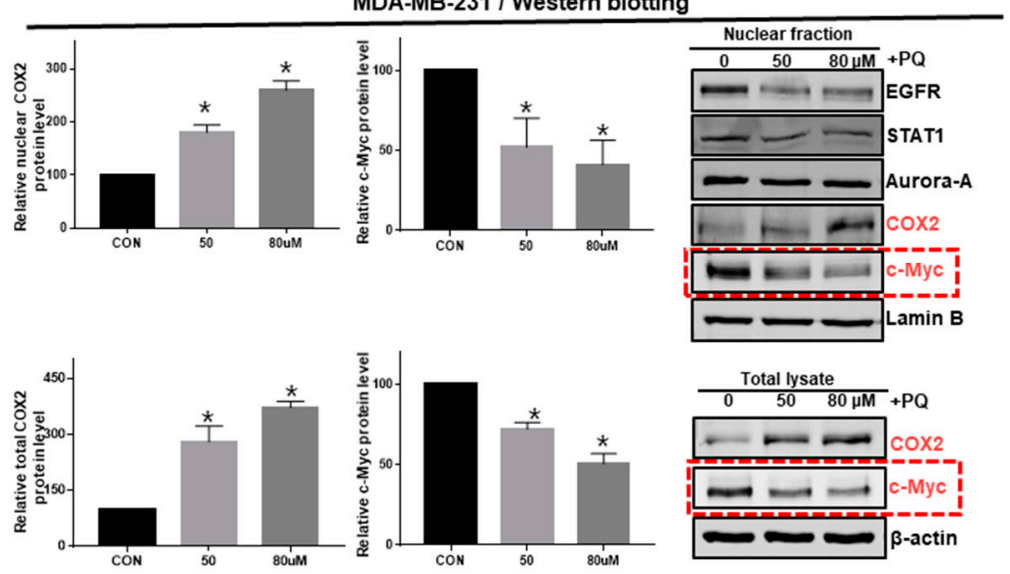

D
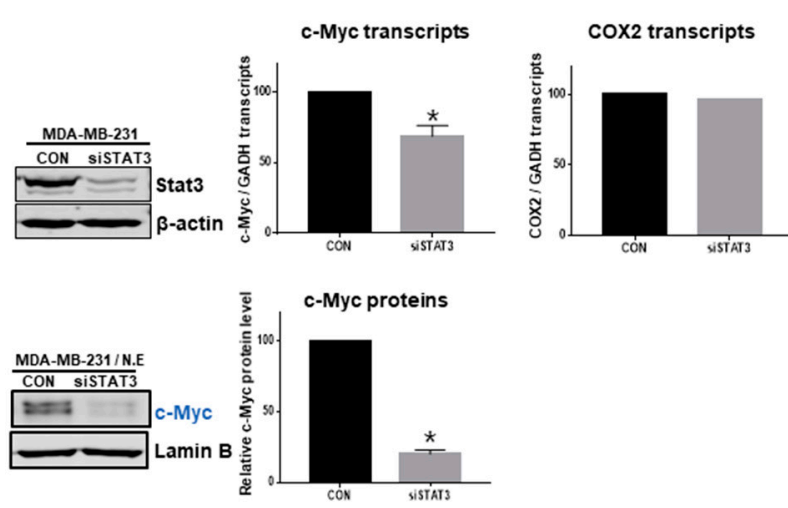

E

;(-)
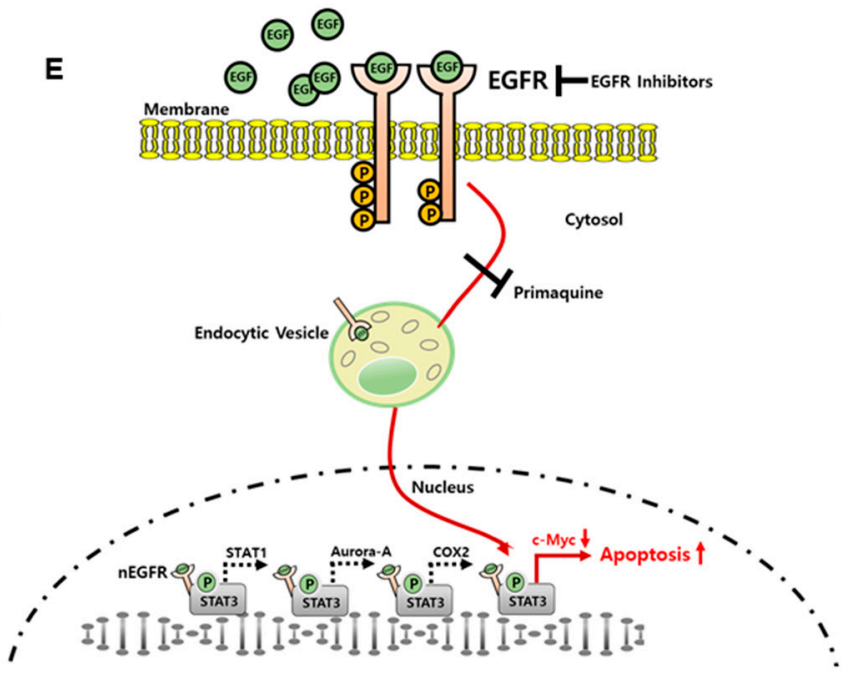

Figure 5. Primaquine reduced the interaction of nEGFR and Stat3 and reduced the transcript and protein levels of c-Myc. (A) nEGFR and Stat3 were immunoprecipitated from the nuclear protein extracts of breast cancer cells under primaquine treatment with anti-EGFR or anti-Stat3 antibodies. Western blotting was performed using the indicated antibodies. (B) The target genes of the Stat3/nEGFR complex were Stat1, Aurora-A, COX2, and c-Myc. (C) Effect of primaquine on transcript levels, total levels, and nuclear levels of the Stat1, Aurora-A, COX2, and c-Myc proteins. Total and nuclear c-Myc protein levels under primaquine $(50$ and $80 \mu \mathrm{M})$ or DMSO treatment conditions. The data are presented as the mean \pm SD; $n=3$; * indicates $p<0.05$ vs. control. (D) siRNA-induced silencing of Stat3 reduced the transcript and protein levels of c-Myc. Representative images of Western blots are shown. Data from triplicate experiments are shown as the mean \pm SD. Compared with control, ${ }^{*}$ indicates $p<0.05$. (E) Inhibition of nEGFR function by primaquine. EGF stimulation induces EGFR dimerization and internalization to endocytic vesicles. EGFR then undergoes nuclear translocation. EGFR is consistently detected in the nuclei of breast cancer cells. nEGFR binds to Stat3, and the EGFR/Stat 3 complex regulates the transcription of Stat1, Aurora kinase A, COX2, and c-Myc. Primaquine reduces nEGFR expression by inhibiting EGFR trafficking. 


\subsection{Primaquine Induces the Apoptosis of Breast Cancer Cells through nEGFR/Stat3-Mediated} $c-M y c$ and $B c l-2$ Downregulation

To examine the mechanism of breast cancer cell death (MDA-MB-231 and HCC-1937) caused by primaquine treatment, we assessed apoptosis. Primaquine induced the early and late apoptosis of breast cancer cells and induced apoptosome formation (Figure 6B,C and Supplementary Figure S1B). We examined the apoptosis-related enzyme caspase- $3 / 7$. Primaquine increased the activity of caspase-3/7 and decreased Bcl-2 levels (Figure 6D,E). Our results showed that primaquine induces the apoptosis of cancer cells by reducing c-Myc and Bcl-2 expression (Figure 6A).

A

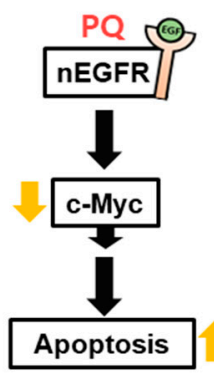

B
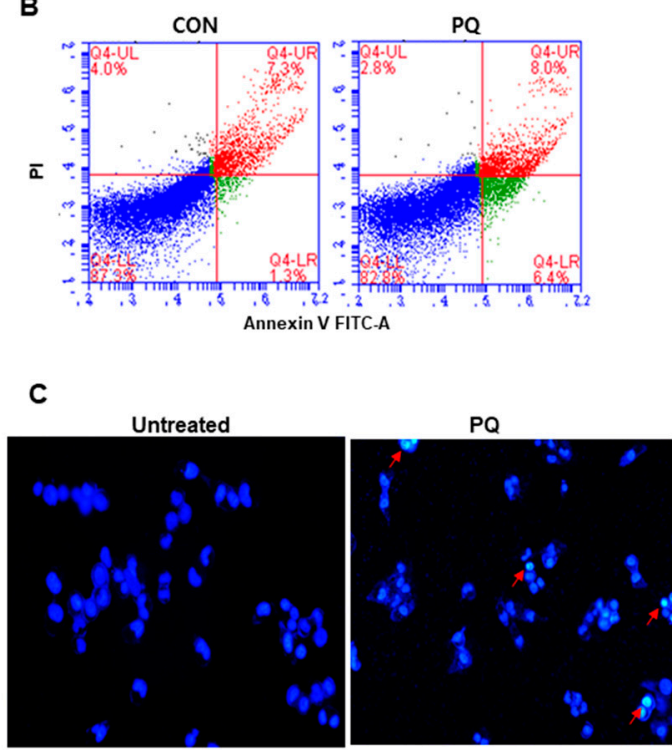

D

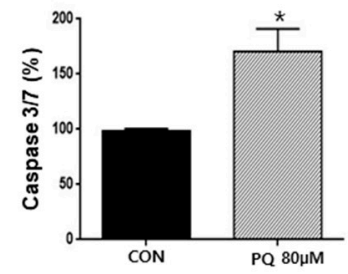

E
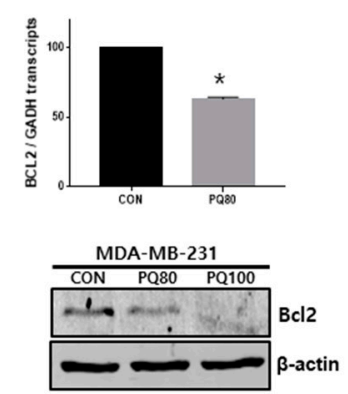

F

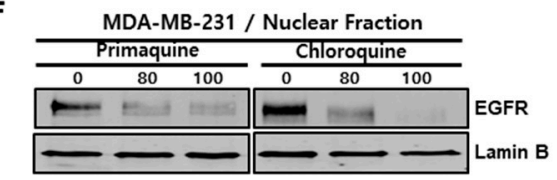

G
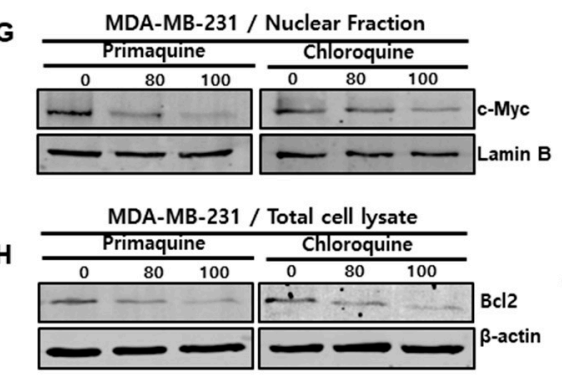
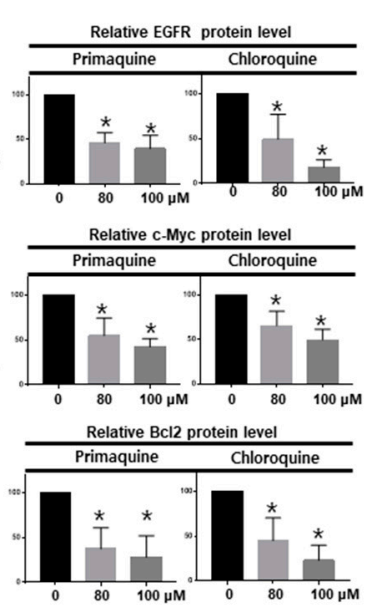

I

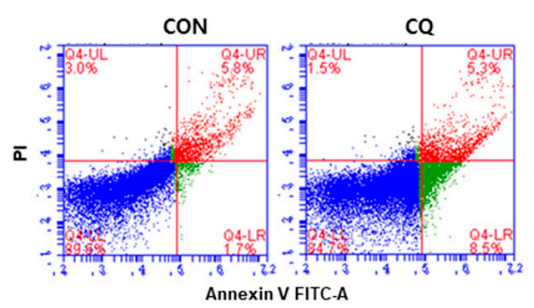

J

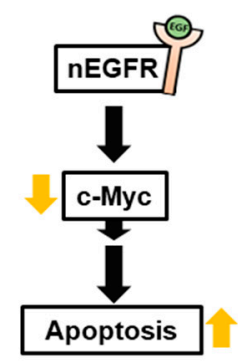

Figure 6. Primaquine and CQ induced the apoptosis of breast cancer through nEGFR and c-Myc regulation. (A) The proposed model for breast cancer cell death induced by primaquine. (B) Primaquine $(80 \mu \mathrm{M})$ induced the apoptosis of cancer cells. Cells undergoing primaquine-induced apoptosis were analyzed by using an annexin V-PI staining kit. (C) Analysis of apoptotic cancer cells by fluorescence staining. The nuclei of breast cancer cells were stained with Hoechst 33258 (magnification, $\times 100$ ). The red arrows indicate apoptotic bodies. (D) The caspase-3/7 activity of breast cancer cells was determined with the Caspase-Gloss $3 / 7$ kit. (E) Effect of primaquine $(0,80$, and $100 \mu \mathrm{M})$ on Bcl-2 protein levels in breast cancer cells. (F-H) Effect of primaquine and CQ $(0,80$, and $100 \mu \mathrm{M})$ on nEGFR, cMyc and Bcl-2 protein levels in breast cancer cells. (I) CQ $(80 \mu \mathrm{M})$ induced the apoptosis of cancer cells. Cells undergoing CQ-induced apoptosis were analyzed by using an annexin V-PI staining kit. (J) The proposed model for CQ-induced breast cancer cell death. The data are presented as the mean $\pm \mathrm{SD} ; n=3 ;{ }^{*}$ indicates $p<0.05$ vs. control. 
As chloroquine $(\mathrm{CQ})$, together with primaquine, is the first-line treatment recommended for malaria and has been reported to promote anticancer activity and regulate the endocytosis-mediated degradation of EGFR [28,30], we assessed the effects of CQ on breast cancer apoptosis. CQ induced the early and late apoptosis of breast cancer cells (Figure 6I). To understand the apoptosis mechanism caused by CQ, we treated breast cancer cells with CQ. CQ and primaquine reduced the protein levels of nEGFR, nuclear c-Myc, and total Bcl-2 (Figure $6 \mathrm{~F}-\mathrm{H}$ ). Our data showed that $\mathrm{CQ}$, similar to primaquine, induces apoptosis through the nEGFR/c-Myc/Bcl-2 pathway (Figure 6J).

\section{Discussion}

The antimalarial drug CQ exerts anti-breast-cancer properties by modulating the microenvironment and inducing apoptosis. CQ may have potential in breast cancer therapy [30]. The antimalarial drug CQ is promising for cancer treatment, and several clinical trials have shown its favorable effects as a novel antitumor drug [31]. Primaquine, sahaquine, and hybrid sahaquine and primaquine dimers also have potential as anticancer agents [32,33]. CQ and hydroxychloroquine have substantial antineoplastic effects in preclinical models and are involved in mechanisms other than autophagy inhibition [34]. Cotreatment with CQ and primaquine sensitized drug-resistant cancer cells through pglycoprotein inhibition [35]. The antineoplastic mechanism of primaquine was previously unclear, but here we have shown a new mechanism of primaquine: it exerts anticancer effects through nEGFR downregulation (Figures 1 and 3). CQ disrupted the endolysosomal system both in vitro and in vivo and induced the disappearance of the early endosome membrane protein EEA1. EGFR levels were decreased under CQ treatment [28]. As primaquine induced the apoptosis of breast cancer, we wanted to identify the apoptotic mechanism of primaquine. We found that primaquine dysregulated the endolysosomal system and EGFR endosomal trafficking, and induced the endocytosis-mediated degradation of EGFR (Figure 2). Our data showed that breast cancer cells express nEGFR and that nEGFR expression was reduced under primaquine treatment (Figures 3 and 5C).

EGFR regulates epithelial tissue development and homeostasis. However, EGFR induces tumorigenesis in lung cancer, breast cancer, and glioblastoma [36]. EGFR functions as a cytoplasmic-membrane-bound RTK that induces growth and survival signals in cancer [6]. Recently, studies have shown that EGFR can be localized to, and function in, the nucleus [11]. nEGFR drives resistance to cetuximab in TNBC. In a breast cancer study, nEGFR was detected in a TNBC cell line and human tumors [5]. In the breast cancer cohort, $40 \%$ of patients showed nEGFR positivity, which was correlated with a worse survival rate $[37,38]$. Research papers have suggested that nEGFR regulates cyclin D1, iNOS, B-Myb, cyclooxygenase-2 (COX-2), aurora kinase A, c-Myc, BCRP, and Stat1 [29]. A previous study showed that the nuclear interaction of Stat3 and EGFR occurs when the iNOS/NO pathway is activated in breast cancer [16]. We assessed the nuclear interaction of Stat3 and EGFR. We confirmed the nuclear interaction of Stat3 and EGFR, and the interaction was reduced under primaquine treatment. Previously, another group showed that the nEGFR/Stat3 complex regulated iNOS, COX-2, aurora kinase A, c-Myc, and Stat1 [11]. Our data showed that primaquine downregulated the c-Myc gene through the regulation of the nEGFR/Stat 3 complex and induced the apoptosis of breast cancer cells (Figures 5 and 6). Exposure of Barrett's and esophageal adenocarcinoma cells to acidic bile salts activated EGFR-Stat3 signaling (EGFR-STAT3 protein complex) and induced the expression of Stat3 target genes (IL-6, IL-17A, BCL-xL, Survivin, and c-MYC) [39]. Our data showed that Stat 3 downregulation using siRNA decreased the expression of the c-Myc gene but not the COX2 gene. Stat3/nEGFR regulated the c-Myc gene and induced apoptosis (Figure 6). Primaquine reduced the interaction of Stat $3 /$ nEGFR and reduced the levels of c-Myc transcripts and proteins. Finally, primaquine induced apoptosis through c-Myc downregulation. c-Myc has a main function in growth regulation, differentiation, and apoptosis [40]. c-Myc regulates important biological pathways involved in the growth and proliferation of tumor cells [41]. c-Myc inhibitors are important therapeutics for cancer. We 
found that primaquine and $C Q$, which are malaria drugs, induce apoptosis through the nEGFR/Stat3 complex and c-Myc downregulation. Taken together, these results indicate that primaquine induces damage to early endosomes and inhibits the nuclear translocation of EGFR. Primaquine inhibits the interaction of Stat3/nEGFR and reduces the transcript and protein levels of c-Myc. Primaquine and CQ induce the apoptosis of breast cancer through nEGFR/Stat3-dependent c-Myc downregulation, which provides a strategy for the treatment of breast cancer by targeting the EGFR signaling components.

\section{Materials and Methods}

\subsection{Reagents and Antibodies}

Primaquine (160393) and chloroquine (CQ; PHR1258) were purchased from Sigma (St. Louis, MO, USA). Anti-EGFR (\#4267), anti-pEGFR (\#2234), anti-pSTAT3 (\#9145), anti-cMyc (\#5605), anti-STAT1 (\#9167), anti-Aurora A (\#14475), and anti-Bcl-2 (\#4223) were obtained from Cell Signaling Technology (Beverly, MA, USA). Anti-STAT3 (SC-482), anti- $\beta$-actin (SC47778), anti-COX2 (SC-19999), and anti-Lamin B (SC-6216) were purchased from Santa Cruz (CA, USA). The antibody for immunofluorescence was anti-EEA1 (610457, BD Transduction Laboratory, Franklin Lakes, NJ, USA).

\subsection{Cell Lines and Media}

Human MDA-MB-231, MCF-7, HCC1937 cells, and MDA-MB-453 breast cancer cells were purchased from the Korean Cell Line Bank (Seoul, Korea). MDA-MB-231 and HCC1937 cells were cultured in Dulbecco's modified Eagle's medium (DMEM, Gibco, Thermo Fisher Scientific, Waltham, MA, USA) supplemented with $10 \%$ fetal bovine serum (Gibco, Thermo Fisher Scientific), penicillin (100 units $/ \mathrm{mL})$, and streptomycin $(100 \mu \mathrm{g} / \mathrm{mL})$ at $37{ }^{\circ} \mathrm{C}$ with $5 \% \mathrm{CO}_{2}$. MDA-MB-231 cells were seeded in a 6-well plate at a density of $2 \times 10^{5}$ cells/well.

\subsection{Cell Proliferation Assay}

We followed a previously reported method [42]. MDA-MB-231, HCC1937 cells, MCF7, and MDA-MB-453 cells were seeded at a density of $1.5 \times 10^{6}$ cells/plate in a 96-well plate and cultured for $24 \mathrm{~h}$, and we treated cells with primaquine for $24 \mathrm{~h}$. We followed the CellTiter $96^{\circledR}$ Aqueous One Solution cell kit protocol and used SpectraMax to read the optical density at $490 \mathrm{~nm}$.

\subsection{Clonogenic and Scratch Assays}

The cells were cultured in a 6-well plate at a density of $1 \times 10^{3}$ cells/well for 1 day and treated with different concentrations of primaquine. The cancer cells were incubated for 7 days, and the colonies were counted. The cells were seeded at a density of $1.5 \times 10^{6}$ cells/plate in a 6-well plate, and scratches were made using a $10-\mu \mathrm{L}$ micropipette tip. After $24 \mathrm{~h}$, the cells were washed with DMEM to remove debris and were then treated with primaquine. We followed a previously described method [43]. The wound areas were photographed under a microscope at $10 \times$ after $18 \mathrm{~h}$.

\subsection{Xenograft Transplantation}

Twelve 4-week-old nude mice were injected with MDA-MB-231 cells and treated with primaquine $(2 \mathrm{mg} / \mathrm{kg})$. Tumor volumes were measured after 90 days using a formula. Mouse experiments were performed as described previously [44]. Animal care and animal experiments were performed in accordance with protocols approved by the Jeju National University Animal Care and Use Committee (JNU-IACUC; Approval Number 2020-035). Female nude mice ( 4 weeks old) were purchased from OrientBio (Seoul, Korea) and kept in mouse facilities for 10 days. 


\subsection{Western Blot Analysis}

Protein samples isolated from breast cancer were separated using $10 \%$ sodium dodecyl sulfate-polyacrylamide gel electrophoresis (SDS-PAGE) and transferred to a PVDF membrane (Millipore, Burlington, MA, USA). Blots were blocked in Odyssey blocking buffer at room temperature for $60 \mathrm{~min}$. The blots were incubated overnight with primary antibodies at $4{ }^{\circ} \mathrm{C}$. After membranes were washed, the blots were incubated with IRDye 680RD and 800W secondary antibodies, and signals were detected using ODYSSEY CLx (Li-Cor, Lincoln, NE, USA).

\subsection{EGFR Degradation and Trafficking}

MDA-MB-231 and MDA-MB-453 cells were cultured in 96-well plates on glass coverslips for EEA1 and EGFR trafficking. MDA-MB-231 cancer cells were treated with $50 \mu \mathrm{M}$ primaquine for $2 \mathrm{~h}$. After treatment, we incubated them for $0,15,30$, or $60 \mathrm{~min}$ with $75 \mathrm{ng} / \mathrm{mL}$ EGF. Finally, we analyzed the samples by immunofluorescence.

\subsection{Immunofluorescence}

We followed a previously reported method [45]. MDA-MB-231and MDA-MB-453 cancer cells were fixed using $4 \%$ paraformaldehyde for $20 \mathrm{~min}$, permeabilized with $0.5 \%$ Triton X-100 for $15 \mathrm{~min}$, blocked with 3\% bovine serum albumin (BSA) for $1 \mathrm{~h}$, stained with primary antibody, and then conjugated to secondary anti-mouse Alexa555 (A32727, Thermo Fisher, Waltham, MA, USA) and rabbit Alexa488 (A32731, Thermo Fisher, Waltham, MA, USA) antibodies. 4',6-Diamidino-2-phenylindole (DAPI) solution was used to stain the nuclei of cancer cells. Finally, EGFR and EEA1 were visualized using a fluorescence microscope (Lionheart FX live cell imager, BioTek).

\subsection{Immunoprecipitation}

MDA-MB-231 cells were washed with $1 \times$ PBS and resuspended in IP lysis buffer. The lysate was incubated with anti-STAT3 and anti-EGFR antibodies for $24 \mathrm{~h}$ at $4{ }^{\circ} \mathrm{C}$. Protein A/G agarose (GenDepot, DAWINbio, Hanam, Korea) was added to the mixtures. All mixtures were centrifuged, washed with IP lysis buffer 3 times, run on SDS-PAGE gels, and subjected to Western blotting.

\subsection{Gene Expression Analysis}

We isolated total RNA from MDA-MB-231 cells. We carried out real-time quantitative RT-PCR using the One Step SYBR PrimeScript RT-PCR kit with SYBR green (Takara, Tokyo, Japan). The primers were purchased from Bioneer Corp. (Daejeon, Korea). PCR was conducted in $10 \mu \mathrm{L}$ of $2 X$ One Step SYBR RT-qPCR Buffer, $1 \mu \mathrm{L}$ PrimeScript One Step Enzyme Mix, $1 \mu \mathrm{g}$ of RNA, and primers for a final volume of $20 \mu \mathrm{L}$ per reaction. We used a previously described method [42].

\subsection{Small Interfering RNA (siRNA)}

To examine the function of c-Myc and STAT3, we treated MDA-MB-231 cells with human c-Myc and STAT3 siRNA. STAT3 siRNA (NM_003150.3, NM_139276.2, NM_213662.1) and c-Myc siRNA (NM_001354870.1, NM_002467.5) were obtained from Bioneer Corp. (Daejeon, Korea). For siRNA transfection, MDA-MB-231 cells were cultured in 6-well plates for 1 day and transfected using Lipofectamine 3000 (Invitrogen, Carlsbad, CA, USA). The level of protein was investigated by Western blotting.

\subsection{Hoechst 33342 Staining and Annexin V/PI Assay}

MDA-MB-231 cells were treated with $20 \mu \mathrm{M}$ primaquine and $50 \mu \mathrm{M}$ CQ for $24 \mathrm{~h}$. After drug treatment, the cells were stained with Hoechst 33342 solution for $30 \mathrm{~min}$ at $37^{\circ} \mathrm{C}$ and then observed using a microscope (Lionheart FX live cell imager, BioTek). Apoptosis assays were performed using an annexin V/propidium iodide (PI) staining kit (BD, San 
Jose, CA, USA). The stained samples were analyzed with an Accuri C6 cytometer (BD, San Jose, CA, USA).

\subsection{Statistical Analysis}

Statistical analysis was performed with GraphPad Prism 7 software (GraphPad Prism Inc., San Diego, CA, USA). All data are shown as the mean \pm standard deviation. Data from three independent experiments were analyzed using a one-way ANOVA. Differences with a p-value less than 0.05 were considered significant.

Supplementary Materials: The following are available online at https:/ /www.mdpi.com/article/10 $.3390 /$ ijms222312961/s1.

Author Contributions: H.-S.C. and J.-H.K. designed this study, participated in all of the experiments, and wrote the manuscript. D.-S.L. wrote the manuscript and supervised the study. All authors have read and agreed to the published version of the manuscript.

Funding: This research was supported by the Basic Science Research Program through the National Research Foundation of Korea (NRF) funded by the Ministry of Education (NRF-2020R1A2C1006316, NRF-2018R1D1A1B07045261, 2020R1A6A3A01100602, and NRF-2016R1A6A1A03012862). This research was supported by the National University Development Project funded by the Ministry of Education (Korea) and the National Research Foundation of Korea (2021). This research was supported by a Korea Basic Science Institute (National Research Facilities and Equipment Center) grant funded by the Ministry of Education (grant No. 2020R1A6C101A188).

Institutional Review Board Statement: All mouse experiments were performed as described previously. Animal care and animal experiments were performed in accordance with protocols approved by the Jeju National University Animal Care and Use Committee (JNU-IACUC; Approval Number 2019-010).

Informed Consent Statement: Informed consent was obtained from all subjects involved in the study.

Data Availability Statement: The data presented in this study are available on request from the corresponding author.

Conflicts of Interest: The authors declare no conflict of interest.

\section{References}

1. Schneider, B.P.; Winer, E.P.; Foulkes, W.D.; Garber, J.; Perou, C.M.; Richardson, A.; Sledge, G.W.; Carey, L.A. Triple-negative breast cancer: Risk factors to potential targets. Clin. Cancer Res. 2008, 14, 8010-8018. [CrossRef]

2. Stevens, K.N.; Vachon, C.M.; Couch, F.J. Genetic susceptibility to triple-negative breast cancer. Cancer Res. 2013, 73, 2025-2030. [CrossRef]

3. Corkery, B.; Crown, J.; Clynes, M.; O'Donovan, N. Epidermal growth factor receptor as a potential therapeutic target in triple-negative breast cancer. Ann. Oncol. 2009, 20, 862-867. [CrossRef]

4. Lehmann, B.D.; Bauer, J.A.; Chen, X.; Sanders, M.E.; Chakravarthy, A.B.; Shyr, Y.; Pietenpol, J.A. Identification of human triple-negative breast cancer subtypes and preclinical models for selection of targeted therapies. J. Clin. Investig. 2011, 121, 2750-2767. [CrossRef] [PubMed]

5. Brand, T.M.; Iida, M.; Dunn, E.F.; Luthar, N.; Kostopoulos, K.T.; Corrigan, K.L.; Wleklinski, M.J.; Yang, D.; Wisinski, K.B.; Salgia, R.; et al. Nuclear epidermal growth factor receptor is a functional molecular target in triple-negative breast cancer. Mol. Cancer Ther. 2014, 13, 1356-1368. [CrossRef]

6. Yarden, Y.; Pines, G. The ERBB network: At last, cancer therapy meets systems biology. Nat. Rev. Cancer 2012, 12, 553-563. [CrossRef] [PubMed]

7. Raymond, E.; Faivre, S.; Armand, J.P. Epidermal growth factor receptor tyrosine kinase as a target for anticancer therapy. Drugs 2000, 60 (Suppl. 1), 15-23. [CrossRef]

8. Wang, Y.N.; Yamaguchi, H.; Hsu, J.M.; Hung, M.C. Nuclear trafficking of the epidermal growth factor receptor family membrane proteins. Oncogene 2010, 29, 3997-4006. [CrossRef]

9. Marti, U.; Burwen, S.J.; Wells, A.; Barker, M.E.; Huling, S.; Feren, A.M.; Jones, A.L. Localization of Epidermal Growth-Factor Receptor in Hepatocyte Nuclei. Hepatology 1991, 13, 15-20. [CrossRef]

10. Brand, T.M.; Iida, M.; Corrigan, K.L.; Braverman, C.M.; Coan, J.P.; Flanigan, B.G.; Stein, A.P.; Salgia, R.; Rolff, J.; Kimple, R.J.; et al. The receptor tyrosine kinase AXL mediates nuclear translocation of the epidermal growth factor receptor. Sci. Signal. 2017, 10, 1064. [CrossRef] [PubMed] 
11. Brand, T.M.; Iida, M.; Li, C.; Wheeler, D.L. The nuclear epidermal growth factor receptor signaling network and its role in cancer. Discov. Med. 2011, 12, 419-432.

12. Du, Y.; Shen, J.; Hsu, J.L.; Han, Z.; Hsu, M.C.; Yang, C.C.; Kuo, H.P.; Wang, Y.N.; Yamaguchi, H.; Miller, S.A.; et al. Syntaxin 6-mediated Golgi translocation plays an important role in nuclear functions of EGFR through microtubule-dependent trafficking. Oncogene 2014, 33, 756-770. [CrossRef] [PubMed]

13. Lo, H.W.; Ali-Seyed, M.; Wu, Y.; Bartholomeusz, G.; Hsu, S.C.; Hung, M.C. Nuclear-cytoplasmic transport of EGFR involves receptor endocytosis, importin beta1 and CRM1. J. Cell Biochem. 2006, 98, 1570-1583. [CrossRef]

14. Wang, Y.N.; Yamaguchi, H.; Huo, L.; Du, Y.; Lee, H.J.; Lee, H.H.; Wang, H.; Hsu, J.M.; Hung, M.C. The translocon Sec61beta localized in the inner nuclear membrane transports membrane-embedded EGF receptor to the nucleus. J. Biol. Chem. 2010, 285, 38720-38729. [CrossRef]

15. Shi, Y.; Tao, Y.; Jiang, Y.; Xu, Y.; Yan, B.; Chen, X.; Xiao, L.; Cao, Y. Nuclear epidermal growth factor receptor interacts with transcriptional intermediary factor 2 to activate cyclin D1 gene expression triggered by the oncoprotein latent membrane protein 1. Carcinogenesis 2012, 33, 1468-1478. [CrossRef] [PubMed]

16. Lo, H.W.; Hsu, S.C.; Ali-Seyed, M.; Gunduz, M.; Xia, W.; Wei, Y.; Bartholomeusz, G.; Shih, J.Y.; Hung, M.C. Nuclear interaction of EGFR and STAT3 in the activation of the iNOS/NO pathway. Cancer Cell 2005, 7, 575-589. [CrossRef]

17. Hanada, N.; Lo, H.W.; Day, C.P.; Pan, Y.; Nakajima, Y.; Hung, M.C. Co-regulation of B-Myb expression by E2F1 and EGF receptor. Mol. Carcinog. 2006, 45, 10-17. [CrossRef] [PubMed]

18. Hung, L.Y.; Tseng, J.T.; Lee, Y.C.; Xia, W.; Wang, Y.N.; Wu, M.L.; Chuang, Y.H.; Lai, C.H.; Chang, W.C. Nuclear epidermal growth factor receptor (EGFR) interacts with signal transducer and activator of transcription 5 (STAT5) in activating Aurora-A gene expression. Nucleic Acids Res. 2008, 36, 4337-4351. [CrossRef]

19. Lo, H.W.; Cao, X.; Zhu, H.; Ali-Osman, F. Cyclooxygenase-2 is a novel transcriptional target of the nuclear EGFR-STAT3 and EGFRvIII-STAT3 signaling axes. Mol. Cancer Res. 2010, 8, 232-245. [CrossRef]

20. Huang, W.C.; Chen, Y.J.; Li, L.Y.; Wei, Y.L.; Hsu, S.C.; Tsai, S.L.; Chiu, P.C.; Huang, W.P.; Wang, Y.N.; Chen, C.H.; et al. Nuclear translocation of epidermal growth factor receptor by Akt-dependent phosphorylation enhances breast cancer-resistant protein expression in gefitinib-resistant cells. J. Biol. Chem. 2011, 286, 20558-20568. [CrossRef]

21. Han, W.; Carpenter, R.L.; Cao, X.; Lo, H.W. STAT1 gene expression is enhanced by nuclear EGFR and HER2 via cooperation with STAT3. Mol. Carcinog. 2013, 52, 959-969. [CrossRef] [PubMed]

22. Liccardi, G.; Hartley, J.A.; Hochhauser, D. EGFR nuclear translocation modulates DNA repair following cisplatin and ionizing radiation treatment. Cancer Res. 2011, 71, 1103-1114. [CrossRef] [PubMed]

23. Li, C.; Iida, M.; Dunn, E.F.; Ghia, A.J.; Wheeler, D.L. Nuclear EGFR contributes to acquired resistance to cetuximab. Oncogene 2009, 28, 3801-3813. [CrossRef]

24. Wheeler, D.L.; Iida, M.; Kruser, T.J.; Nechrebecki, M.M.; Dunn, E.F.; Armstrong, E.A.; Huang, S.; Harari, P.M. Epidermal growth factor receptor cooperates with Src family kinases in acquired resistance to cetuximab. Cancer Biol. Ther. 2009, 8, 696-703. [CrossRef]

25. Iida, M.; Brand, T.M.; Campbell, D.A.; Li, C.; Wheeler, D.L. Yes and Lyn play a role in nuclear translocation of the epidermal growth factor receptor. Oncogene 2013, 32, 759-767. [CrossRef]

26. Le Roy, C.; Wrana, J.L. Clathrin- and non-clathrin-mediated endocytic regulation of cell signalling. Nat. Rev. Mol. Cell Biol. 2005, 6, 112-126. [CrossRef]

27. Jo, U.; Park, K.H.; Whang, Y.M.; Sung, J.S.; Won, N.H.; Park, J.K.; Kim, Y.H. EGFR endocytosis is a novel therapeutic target in lung cancer with wild-type EGFR. Oncotarget 2014, 5, 1265-1278. [CrossRef]

28. Mauthe, M.; Orhon, I.; Rocchi, C.; Zhou, X.; Luhr, M.; Hijlkema, K.J.; Coppes, R.P.; Engedal, N.; Mari, M.; Reggiori, F. Chloroquine inhibits autophagic flux by decreasing autophagosome-lysosome fusion. Autophagy 2018, 14, 1435-1455. [CrossRef]

29. Brand, T.M.; Iida, M.; Luthar, N.; Starr, M.M.; Huppert, E.J.; Wheeler, D.L. Nuclear EGFR as a molecular target in cancer. Radiother. Oncol. 2013, 108, 370-377. [CrossRef]

30. Zhang, Y.; Cao, Y.; Sun, X.; Feng, Y.; Du, Y.; Liu, F.; Yu, C.; Jin, F. Chloroquine (CQ) exerts anti-breast cancer through modulating microenvironment and inducing apoptosis. Int. Immunopharmacol. 2017, 42, 100-107. [CrossRef] [PubMed]

31. Sotelo, J.; Briceno, E.; Lopez-Gonzalez, M.A. Adding chloroquine to conventional treatment for glioblastoma multiforme: A randomized, double-blind, placebo-controlled trial. Ann. Intern. Med. 2006, 144, 337-343. [CrossRef] [PubMed]

32. Pavic, K.; Rubinic, B.; Rajic, Z.; Fontinha, D.; Prudencio, M.; Uzelac, L.; Kralj, M.; Held, J.; Zorc, B. Primaquine homodimers as potential antiplasmodial and anticancer agents. Bioorg. Med. Chem. Lett. 2019, 29, 126614. [CrossRef]

33. Beus, M.; Rajic, Z.; Maysinger, D.; Mlinaric, Z.; Antunovic, M.; Marijanovic, I.; Fontinha, D.; Prudencio, M.; Held, J.; Olgen, S.; et al. SAHAquines, Novel Hybrids Based on SAHA and Primaquine Motifs, as Potential Cytostatic and Antiplasmodial Agents. Chem. Open 2018, 7, 624-638. [CrossRef]

34. Manic, G.; Obrist, F.; Kroemer, G.; Vitale, I.; Galluzzi, L. Chloroquine and hydroxychloroquine for cancer therapy. Mol. Cell Oncol. 2014, 1, e29911. [CrossRef]

35. Kim, J.H.; Choi, A.R.; Kim, Y.K.; Yoon, S. Co-treatment with the anti-malarial drugs mefloquine and primaquine highly sensitizes drug-resistant cancer cells by increasing P-gp inhibition. Biochem. Biophys. Res. Commun. 2013, 441, 655-660. [CrossRef]

36. Sigismund, S.; Avanzato, D.; Lanzetti, L. Emerging functions of the EGFR in cancer. Mol. Oncol. 2018, 12, 3-20. [CrossRef] [PubMed] 
37. Lo, H.W.; Xia, W.; Wei, Y.; Ali-Seyed, M.; Huang, S.F.; Hung, M.C. Novel prognostic value of nuclear epidermal growth factor receptor in breast cancer. Cancer Res. 2005, 65, 338-348. [PubMed]

38. Hadzisejdic, I.; Mustac, E.; Jonjic, N.; Petkovic, M.; Grahovac, B. Nuclear EGFR in ductal invasive breast cancer: Correlation with cyclin-D1 and prognosis. Mod. Pathol. 2010, 23, 392-403. [CrossRef]

39. Bhat, A.A.; Lu, H.; Soutto, M.; Capobianco, A.; Rai, P.; Zaika, A.; El-Rifai, W. Exposure of Barrett's and esophageal adenocarcinoma cells to bile acids activates EGFR-STAT3 signaling axis via induction of APE1. Oncogene 2018, 37, 6011-6024. [CrossRef]

40. Hoffman, B.; Liebermann, D.A. Apoptotic signaling by c-MYC. Oncogene 2008, 27, 6462-6472. [CrossRef]

41. Elbadawy, M.; Usui, T.; Yamawaki, H.; Sasaki, K. Emerging Roles of C-Myc in Cancer Stem Cell-Related Signaling and Resistance to Cancer Chemotherapy: A Potential Therapeutic Target Against Colorectal Cancer. Int. J. Mol. Sci. 2019, 20, 2340. [CrossRef] [PubMed]

42. Choi, H.S.; Kim, J.H.; Kim, S.L.; Deng, H.Y.; Lee, D.; Kim, C.S.; Yun, B.S.; Lee, D.S. Catechol derived from aronia juice through lactic acid bacteria fermentation inhibits breast cancer stem cell formation via modulation Stat3/IL-6 signaling pathway. Mol. Carcinog. 2018, 57, 1467-1479. [CrossRef] [PubMed]

43. Choi, H.S.; Kim, S.L.; Kim, J.H.; Deng, H.Y.; Yun, B.S.; Lee, D.S. Triterpene Acid (3-O-p-Coumaroyltormentic Acid) Isolated From Aronia Extracts Inhibits Breast Cancer Stem Cell Formation through Downregulation of c-Myc Protein. Int. J. Mol. Sci. 2018, 19, 2528. [CrossRef] [PubMed]

44. Kim, S.L.; Choi, H.S.; Kim, J.H.; Jeong, D.K.; Kim, K.S.; Lee, D.S. Dihydrotanshinone-Induced NOX5 Activation Inhibits Breast Cancer Stem Cell through the ROS/Stat3 Signaling Pathway. Oxid. Med. Cell Longev. 2019, 2019, 9296439. [CrossRef]

45. Kim, J.H.; Choi, H.S.; Kim, S.L.; Lee, D.S. The PAK1-Stat3 Signaling Pathway Activates IL-6 Gene Transcription and Human Breast Cancer Stem Cell Formation. Cancers 2019, 11, 1527. [CrossRef] 